\title{
Application of alkynyliodonium salts to natural products synthesis: a mini-review of recent work at Penn State
}

\author{
Ken S. Feldman \\ Department of Chemistry, The Pennsylvania State University, University Park, PA 16802 USA \\ E-mail: ksf@chem.psu.edu
Dedicated with fondness to Professor Anastasios Varvoglis on the occasion of his $65^{\text {th }}$ birthday \\ (received 09 Jan 03; accepted 20 June 03; published on the web 24 June 03)

\begin{abstract}
Total syntheses of the polycyclic natural products agelastatin $\mathrm{A}$ and pareitropone have been accomplished through the agency of alkynyliodonium salts. A review of these efforts, which emphasizes the value of hypervalent iodine chemistry in raising the molecular complexity of substrates upon chemical transformation, follows.
\end{abstract}

Keywords: Agelastatin A, pareitropone, alkynyliodonium salts, natural products synthesis

A desire to exploit the unique reactivity patterns offered by alkynyliodonium salts (5) has fueled a continuing effort to develop efficient syntheses of naturally occurring principles at Penn State. A progress report that details some recently completed projects in the areas of agelastatin A (1) and pareitropone (2) assembly is described herein. ${ }^{1}$ These advances owe much to the seminal contributions of many workers around the world, especially those recorded by the groups of Stang, Koser and Moriarity in the US, Ochiai and Kita in Japan, and, of course, by Anastasios Varvoglis' group at Thessaloniki. ${ }^{2,3,4}$ In particular, this renaissance in hypervalent iodine chemistry is captured beautifully in two recent scholarly treatises authored by Varvoglis. ${ }^{3}$ These books provide the foundation of, and the inspiration for, the synthesis work outlined below.

Successful execution of total synthesis efforts often hinges on identifying solutions to problems of chemo- and stereoselectivity. Synthesis routes featuring alkynyliodonium salts are no exception, and key discoveries by Stang, ${ }^{5}$ and independently, Ochiai, ${ }^{6}$ have provided insight into how these types of selectivity issues might be addressed. In 1991, Stang and Zhdankin introduced PhI(CN)OTf (4) as a phenyliodonium transfer reagent of exquisite chemoselectivity in preparing alkynyliodonium units 5 from alkynylstannane precursors $\mathbf{3}$, Scheme 1 . $^{5}$ Prior to this advance, it was unclear whether any of the existing iodonium transfer reagents could perform with the requisite selectivity that reaction with complex, multifunctional substrates demanded. 
This issue of chemoselectivity became acute when several ongoing projects required generation of alkynyliodonium species in the presence of tethered nucleophiles such as alcohols, amides, imides, carbamates, alkenes, etc., in order to reach synthesis objectives. As a practical matter, the Stang reagent operated with the necessary selectivity where some other iodonium transfer reagents were less satisfactory.

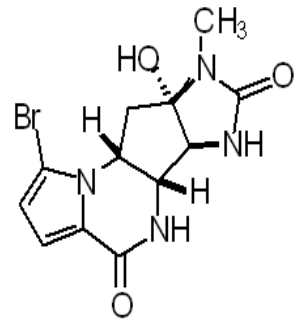

1 agalastatin $A$

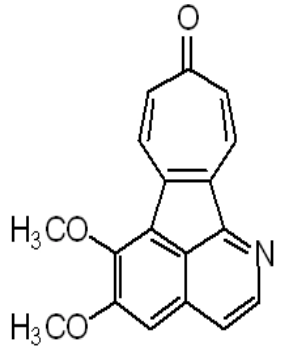

2 pareitropone
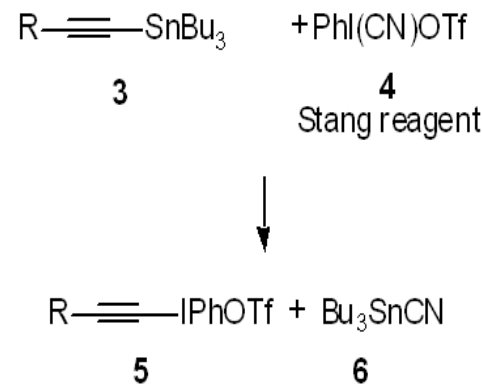

5

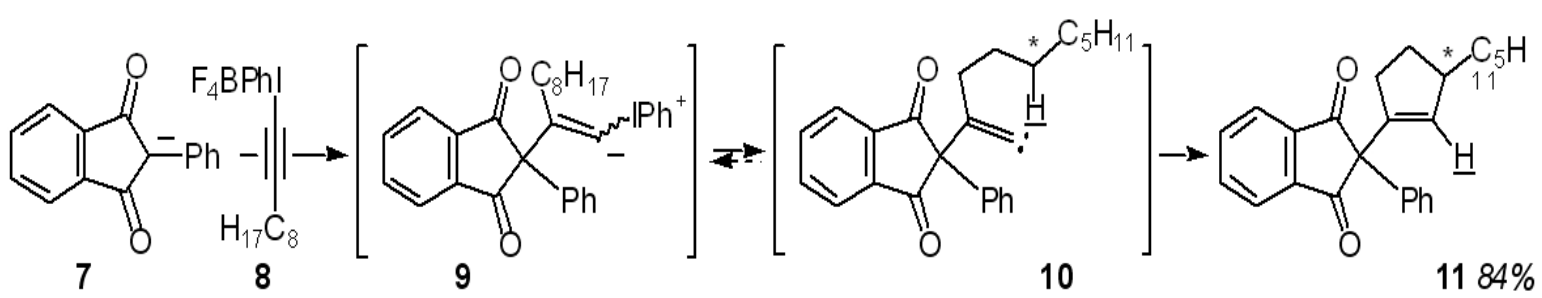

\section{Scheme 1}

The question of stereoselectivity upon formation of $\mathrm{sp}^{2}-\mathrm{sp}^{3} \mathrm{C}-\mathrm{C}$ bonds was central to the agelastatin A project. This key C-C bond-forming step was envisioned to proceed in a manner suggested from the original work of Ochiai, ${ }^{6}$ who showed in 1986 that alkynyliodonium salts were competent electrophiles when partnered with select soft nucleophiles to provide cyclopentene products $\mathbf{7} \rightarrow \mathbf{1 1}$, Scheme 1 . This remarkable transformation encompasses the formation of two new C-C bonds and one new C-H bond with complete retention of stereochemistry at the newly formed stereogenic center. In addition, bond formation at an otherwise unactivated $\mathrm{C}-\mathrm{H}$ position $(*)$, presumably via the agency of an unobserved alkylidenecarbene (10), speaks to the value of this process in raising the molecular complexity of the substrate upon reaction. Thus, with knowledge of a chemoselective iodinating reagent and a stereoselective C-C bond forming process, total syntheses of the cognate targets agelastatin A and pareitropone were undertaken.

The central theme of these synthesis efforts emerges from consideration of the dual reactivity patterns available to alkynyliodonium salts; they are in turn electrophilic and then carbenic in character, and this reaction profile can be utilized in orthogonal bond forming processes to prepare structurally complex products from relatively simple substrates. Alkynyliodonium salts serve as "bottled" alkylidenecarbenes in this context. There are several methods for generating 
this reactive carbene intermediate (Scheme 2), ${ }^{7}$ but only the iodonium salt approach offers the advantage of convergency upon $\mathrm{R}_{1}$ and $\mathrm{R}_{2}$ introduction. All other methods require construction of a substrate already bearing both $\mathrm{R}_{1}$ and $\mathrm{R}_{2}$ substituents.

The chemistry of alkylidenecarbenes has been developed by many groups using a variety of experimental and computational strategies, ${ }^{8}$ Scheme 2 . The carbene itself appears to be a singlet species that features a lone pair of electrons in an sp-hybridized orbital and an empty p-orbital that renders the species mildly electrophilic and very electron deficient. A combination of calculations and spectroscopic observation on the parent species vinylidene $\left(\mathrm{H}_{2} \mathrm{C}=\mathrm{C}\right.$ :) suggests a heat of formation of $100 \mathrm{kcal} / \mathrm{mol}^{8}{ }^{8 \mathrm{f}}$ marking this 2-carbon species as one of the most energetic organic fragments known. This high energy is discharged through a variety of pathways that all take advantage of the empty p orbital to provide kinetically accessible decomposition routes. 1,2Shift of one of the carbene's substituents, 1,5 C-H insertion (cf. Ochiai), alkene addition, and heteroatom-lone pair capture are among the processes that are detected as part of the synthesis studies described shortly. As will be documented in these studies, judicious placement of carbene traps and/or electronic perturbation of reactivity are necessary to steer this exceedingly reactive carbene intermediate down a preferred pathway to achieve a desired synthesis objective.

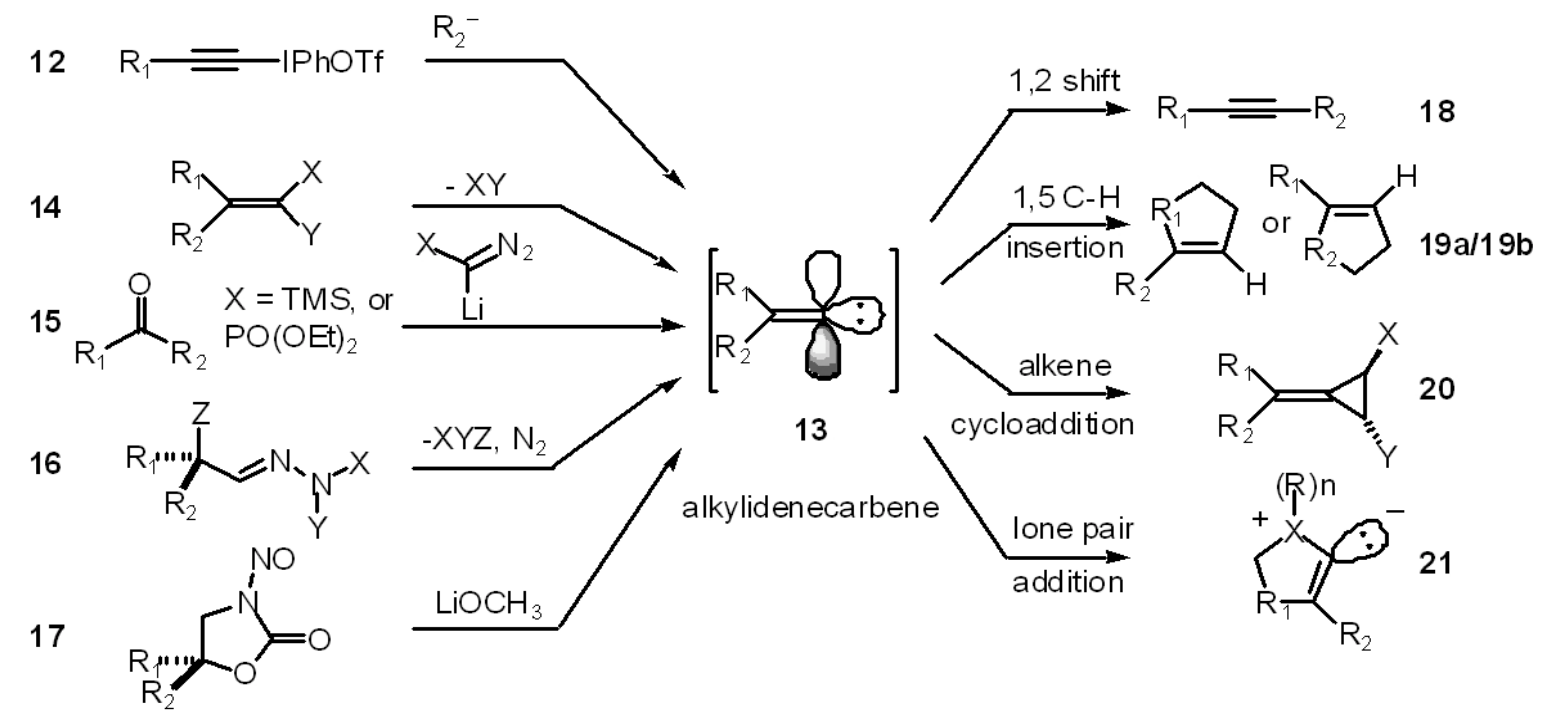

\section{Scheme 2}

The inaugural project in natural products synthesis via alkynyliodonium salt chemistry targeted the tetracyclic marine principle agelastatin A (1), Scheme 3. ${ }^{\text {a }}$ This sponge metabolite features a cyclopentane ring bearing four stereogenic centers, a molecular fragment potentially accessible through the cyclopentene-forming alkynyliodonium salt chemistry of Ochiai. The introduction of four nitrogen substituents into this compact core will require careful staging of the $\mathrm{C}-\mathrm{N}$ bond forming sequences in order to avoid a situation where the presence of too many inductively electron depleting substituents on the cyclopentane nucleus suppresses further 
electrophilic reactivity. Again, the alkynyliodonium salt $\rightarrow$ alkylidenecarbene $\rightarrow$ cyclopentene sequence provides a framework to address this concern, as the resulting functionality raises the ring's oxidation level to the extent that subsequent $\mathrm{C}-\mathrm{N}$ bond formations are "downhill" in energy, vide infra. A first generation approach to the agelastatin target $\mathbf{1}$ might proceed through the cyclopentene 27, a species anticipated to be accessible from alkylidenecarbene 26a and alkynyliodonium salt 25. ${ }^{9}$ The cyclization substrate $\mathbf{2 4}$ was prepared in a straightforward manner from Garner's aldehyde (22), taking advantage of Fujisawa's chelation controlled imine addition methodology ${ }^{10}$ to set the desired stereochemistry between $\mathrm{C}(4)$ and $\mathrm{C}(8)$ in 23 (agelastatin A numbering). Conversion of the polyfunctional alkynylstannane $\mathbf{2 4}$ into the iodonium salt 25 emphasized the value of the Stang reagent's selectivity for alkyne-tin bonds. Attempted intramolecular bicyclization of the urea imide 25 was modeled on Ochiai's guiding stabilized enolate/alkynyliodonium salt work, and the acquisition of a bicyclic alkene-containing product generated initial optimism about this approach. However, a more careful examination of this species' spectral data revealed it to be something else entirely, the dihydropyrrole 29. A mechanistic description of this process suggests where the desired chemistry was derailed. Apparently, the putative alkylidenecarbene intermediate $\mathbf{2 6}$ chooses to add to the BOC carbamate's lone pair rather than participate in the desired 1,5 C-H insertion. In retrospect, perhaps it is not that surprising that even a BOC carbamate's nitrogen possesses more accessible electron density than an unactivated $\mathrm{C}-\mathrm{H}$ bond, but at the time this result was completely unexpected. This initial pass at an alkynyliodonium salt-based route to agelastatin A could go no further, but the effort did provide the valuable lesson that appropriately positioned heteroatom lone pairs must be considered when assessing the reactivity options available to electrophilic alkylidenecarbenes.

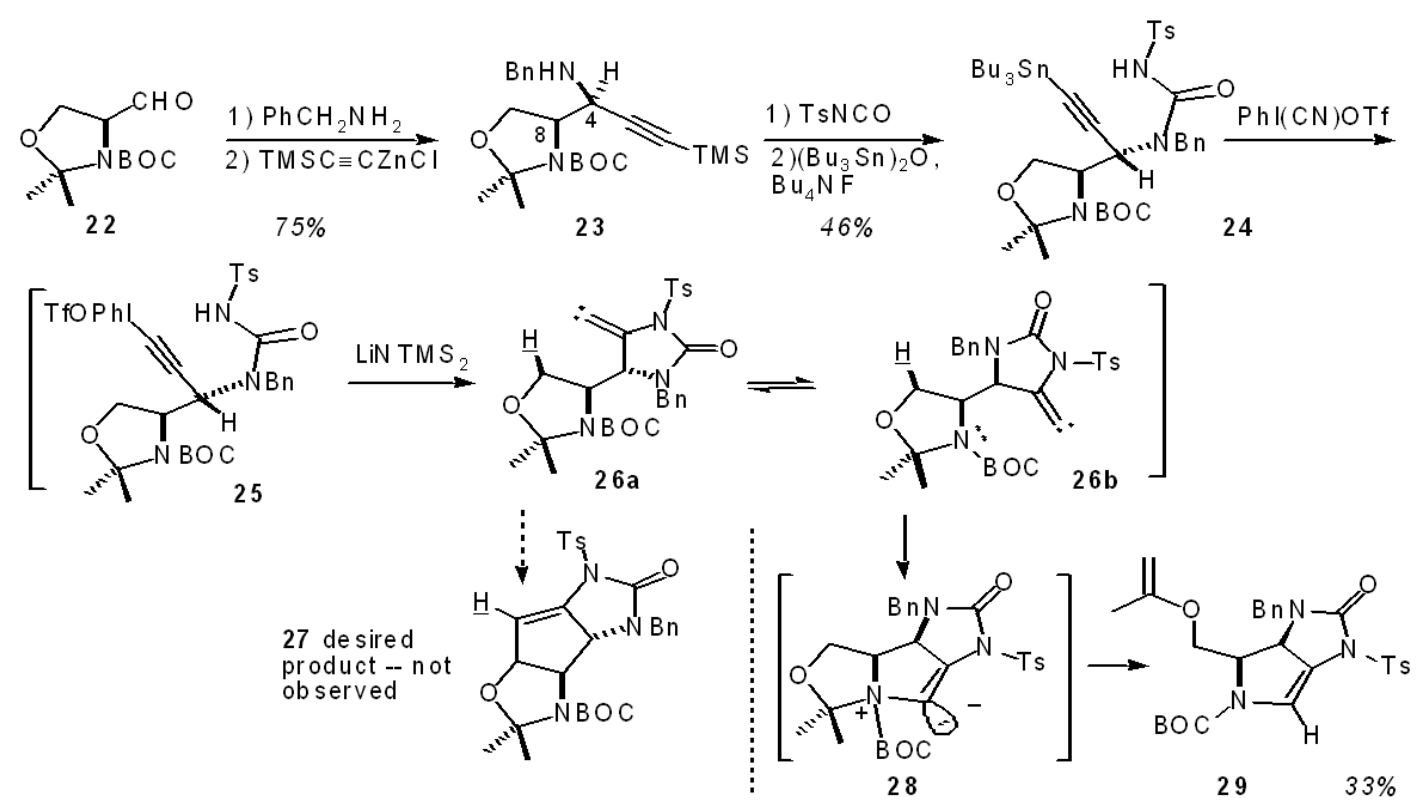

\section{Scheme 3}


A second-generation study began with these caveats in mind, Scheme 4. In this modification of the agelastatin A synthesis route, a deliberate exploration of substituent effects on the competition between 1,5 C-H insertion (31a $\rightarrow$ 30, desired) and O-lone pair addition (from 31b, undesired) for an intermediate alkylidenecarbene was given high priority. Towards this end, a series of related alkynylstannanes 33, 37, 42, and 46 were prepared, Scheme 5. Treatment of each of these substrates, independently, with PhI(CN)OTf and then the exogenous nucleophile $p$ $\mathrm{TolSO}_{2} \mathrm{Na}$ led to rapid formation of a suite of cyclic products. The ketal-containing species 33 was a particularly poor substrate for this reaction, and only trace amounts of characterizable products 35 and 36 could be isolated after extensive chromatography. Analysis of these components suggested that carbene-oxygen lone pair combination held sway over $\mathrm{C}$-H insertion by a 3:1 margin, although the yields were so low that no undue importance should be attached to these numerical values. Increasing the electron demand on the target oxygen in substrate $\mathbf{3 7}$ should foster a proportionate decrease in electron density on that atom, and the electrophilic carbene 38 now was diverted to the $\mathrm{C}-\mathrm{H}$ insertion option 39 with some preference. The alkyl (benzyl) residue in $\mathbf{3 8}$ can promote significant electron donation from $\mathrm{N}$ to $\mathrm{CO}$, a situation that might countermand the desired $\mathrm{O} \rightarrow \mathrm{CO}$ electron donation. In this circumstance, the target oxygen apparently retains sufficient electron density to react with the alkylidenecarbene through a now minor reaction channel to yield 40. This undesired oxygen lone pair addition was suppressed completely when the electron contribution of the ring nitrogen was minimized by attachment of a second carbonyl, cf. 42 and 46. With these substrates, no carbene-oxygen trapping products were detected at all, a result that likely stemmed from the now more compelling electron demand of the ring carbonyl. Thus, by rational choice of oxygen substituent, the competition between $\mathrm{C}-\mathrm{H}$ insertion and oxygen lone pair addition can be directed down the desired channel. However, the overall chemical yields of C-H insertion products $\mathbf{4 4}$ and $\mathbf{4 8}$ had not enjoyed a corresponding increase. Apparently, a third process available to the intermediate alkylidenecarbene now has intervened. Further examination of the crude reaction mixtures derived from $\mathbf{4 2}$ and $\mathbf{4 6}$ led to isolation of the alkynylsulfone-containing products $\mathbf{4 5}$ and $\mathbf{4 9}$, respectively. The formation of $\mathbf{4 9}$ as the major reaction product from $\mathbf{4 6}$ indicated that at least for the alkylidenecarbene intermediate 47, the 1,2-shift option (cf. Scheme 2) holds dominion. Labeling studies conducted by Ochiai in a related but simpler case revealed that it is the sulfone moiety, and not the alkyl residue, which migrates. ${ }^{11}$ Extrapolation from this earlier work to 43 or 47 may or may not be warranted. So, it seems inescapable that packing so many inductively electron withdrawing groups in the vicinity of the scissile $\mathrm{C}-\mathrm{H}$ bond in $\mathbf{4 3}$ or $\mathbf{4 7}$ depletes its electron density to such an extent that the normally slower sulfone migration can now compete with $\mathrm{C}-\mathrm{H}$ insertion chemistry. Thus, a fine balance must be struck between too much and too little electron demand along the molecular framework in order to give the desired $\mathrm{C}$-H insertion chemistry a chance to be expressed. Even though the chemical yield of the desired cyclopentene 48 was disappointing, enough material was in hand to continue the agelastatin A synthesis plan. 


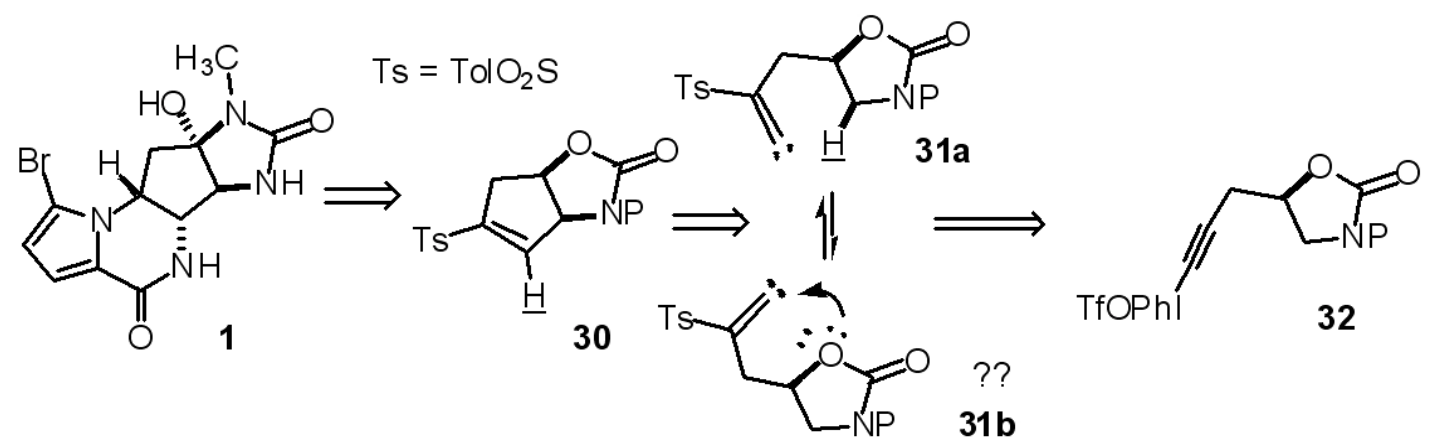

\section{Scheme 4}
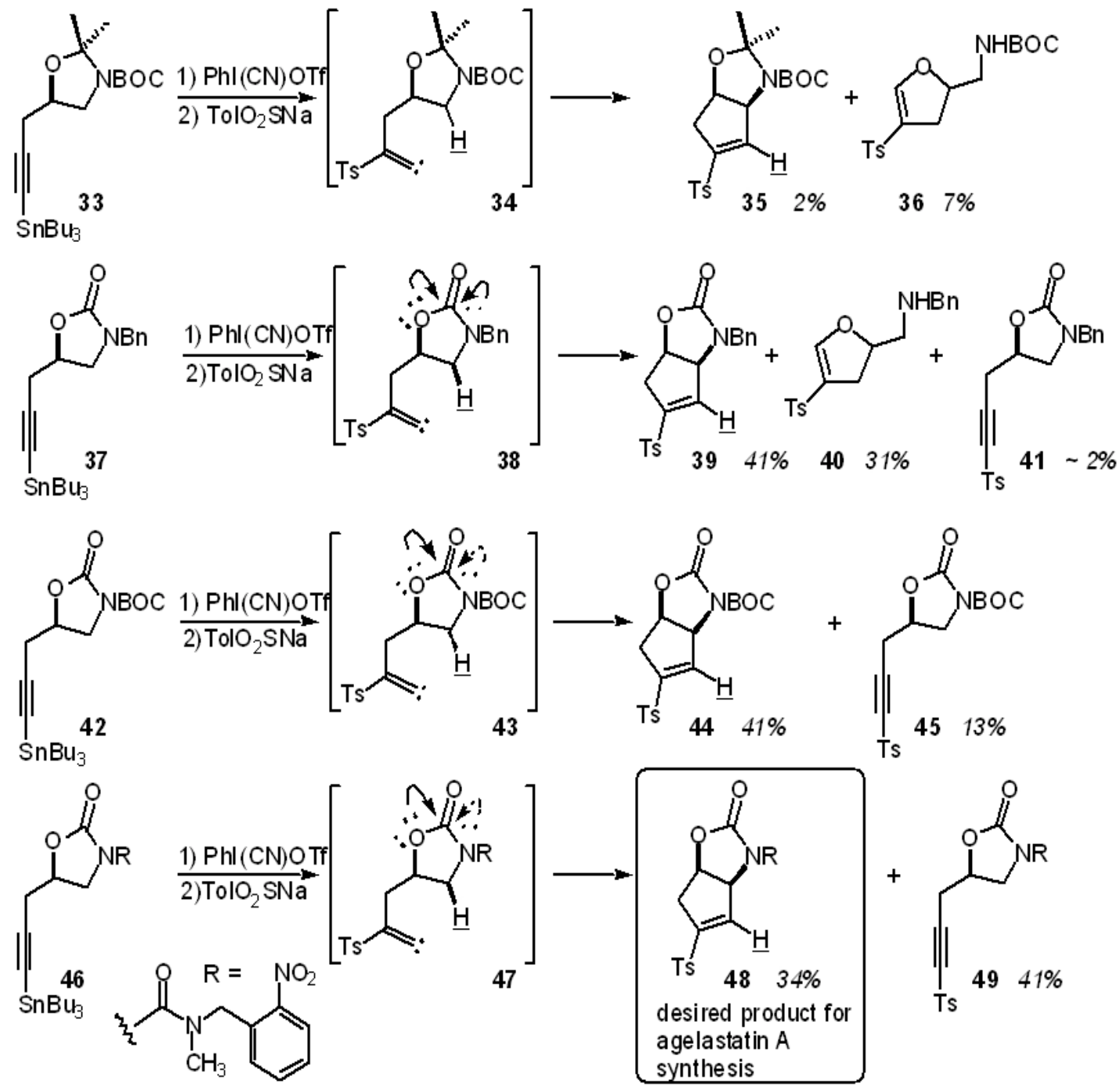

\section{Scheme 5}


The next challenges involved elaboration of the remaining cyclopentane functionality in a stereo- and regioselective manner, Scheme 6 . The second ring nitrogen could be introduced in protected form with complete selectivity by taking advantage of the activating effect of the sulfone moiety and by using the steric bias of the convex shape of bicycle 48. Acylation of the secondary amine product with pyrrole 2-carboxylic acid chloride and subsequent hydrolytic rupture of the product's oxazolidinone ring delivered cyclopentanol $\mathbf{5 0}$ in good yield. This cyclopentanol ring features the pivotal trans relationship between the $C(4)$ and $C(8)$ nitrogen substituents required for agelastatin A. Subjection of this cyclopentanol to Swern oxidation conditions triggered a cascade of reactions that ultimately afforded the tricycle 52, a species that finally begins to resemble the target structure. This multi-step transformation utilizes the sulfone moiety in its third and final role as it serves as a leaving group to generate a reactive intermediate enone 51. In a separate series of experiments, use of less basic oxidation reaction conditions (e.g., Dess Martin) led to identification of enone $\mathbf{5 1}$ in the crude reaction mixture. Treatment of this crude enone with $\mathrm{Et}_{3} \mathrm{~N}$ rapidly yielded the tricycle 52 .

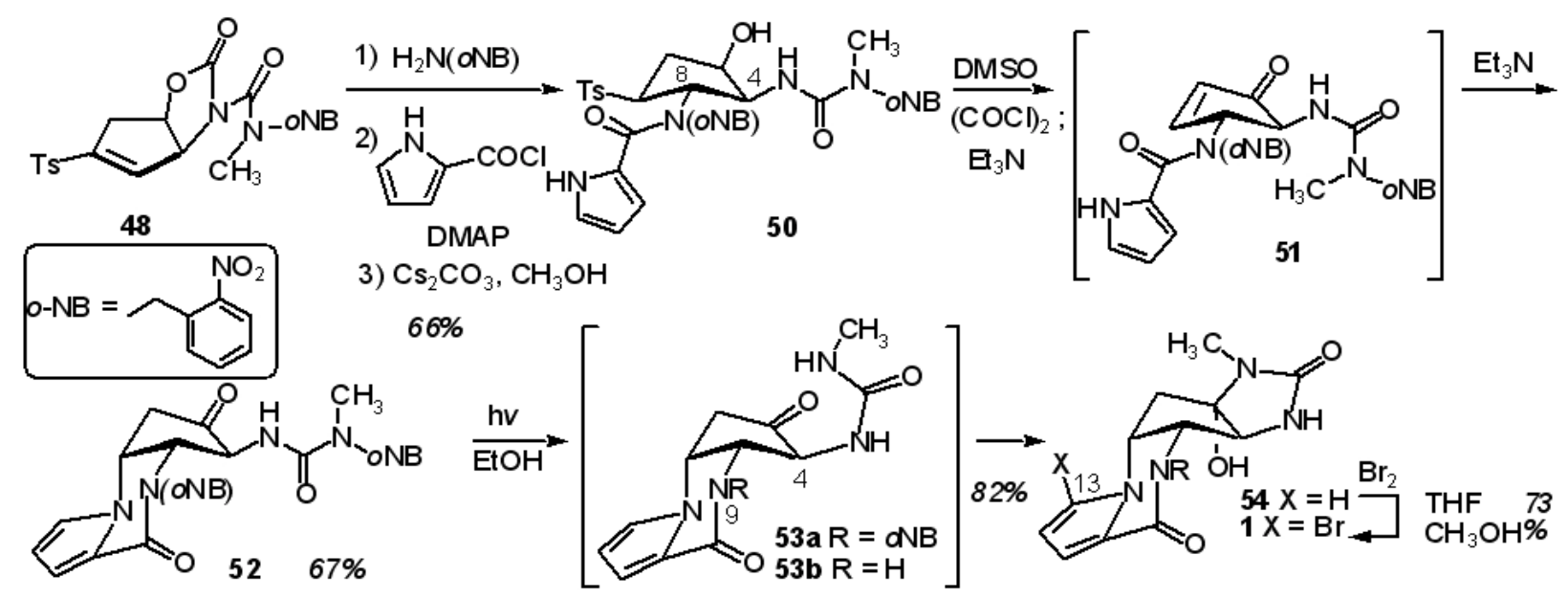

\section{Scheme 6}

The completion of the agelastatin A work required two operations of note: bis $N$-deprotection within 52, and selective monobromination of the pyrrole product at C(13), Scheme 6. Photolytic removal of the $\boldsymbol{o}$-nitrobenzyl units in $\mathbf{5 2}$ furnished the tetracyclic debromoagelastatin structure $\mathbf{5 4}$, presumably via the unobserved open chain intermediates 53a and/or 53b. Interestingly, prior scouting experiments with alternative protecting groups revealed that an $\mathrm{N}(9)$ unprotected cyclopentanone similar to $\mathbf{5 3 b}$ suffered facile epimerization at C(4). That $\mathbf{5 4}$ was not formed as a

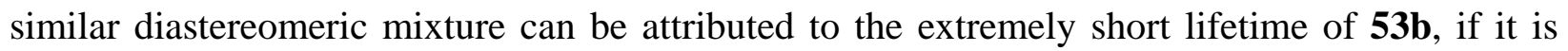
formed at all. Monobromination of the pyrrole ring within 54 benefited from some precedent, ${ }^{12}$ but with this particular substrate, significant optimization of experimental parameters was required to obtain $\mathbf{1}$ in good yield and free of isomers. Eventually, electrophilic and not radical 
bromination conditions in a polar reaction medium sufficed to generate the desired target agelastatin A (1) in high yield. Overall, it is clear that alkynyliodonium salt chemistry can be used advantageously to deliver functionally rich cyclopentene intermediates from relatively simple and accessible substrates, a hallmark of efficient synthesis design.

The second target of opportunity for alkynyliodonium salt chemistry was the tropoloisoquinoline alkaloid pareitropone (2), Scheme 7. Upon cursory inspection, it is less than obvious that a connection can be made between the tetracyclic and fully aromatic skeleton of $\mathbf{2}$ and any of the products of alkynyliodonium salt/alkylidenecarbene chemistry charted in Scheme 2. However, an unanticipated result upon exploration of some routine indole formation chemistry provided the conceptual refocusing necessary to connect 2 to alkynyliodonium salt chemistry. ${ }^{13}$ Exposure of tosylanilide (55) to base and then propynyl(phenyl)iodonium triflate furnished the expected indole product $\mathbf{5 7}$ via the presumed intermediacy of alkylidenecarbene $\mathbf{5 6}$. However, lesser amounts of a highly colored material whose spectral characterization led to the structural assignment 59 were identified as well. Mechanistic speculation ran to the series of intermediates 56b $\rightarrow 58$ en route to 59 from 55. This process demonstrated that appropriately positioned aryl rings are sufficiently electron rich to trap tethered alkylidenecarbenes, even if highly strained products result (58 formally includes a trans alkene in a six-membered ring). Discharge of this strain through electrocyclization provides a productive decomposition pathway to 59. Therefore, the well known alkylidenecarbene-alkene addition reaction can be extended to aryl rings leading to cycloheptatriene products, provided that lower energy options like 1,5 C-H insertions are not available. The resemblance of $\mathbf{5 9}$ to $\mathbf{2}$ now makes the application of this sequence of reactions to pareitropone more explicable. The target $\mathbf{2}$ might arise from further oxidation of the dihydroisoquinoline precursor 60, which in turn might be derived from iodonium salt chemistry triggered by intramolecular nucleophilic addition within 63. Successful implementation of this sequence requires selective iodination of the alkyne function in the presence of the mildly nucleophilic tosylanilide and aryloxysilane moieties, a level of chemoselectivity that should fall within the purview of the Stang reagent. A more compelling issue of selectivity arises upon consideration of the decomposition options available to exocyclic carbene 62. 1,2-Shifts are unlikely due to the incipient strain of the putative cycloheptyne product, but 1,6 C-H insertion to afford phenanthrene $\mathbf{6 4}$ cannot be readily dismissed. Earlier work by Harrington $(\mathbf{6 5} \rightarrow \mathbf{6 6})^{14}$ and also more recent work from Penn State $(\mathbf{6 8} \rightarrow \mathbf{6 9})^{15}$ provides ample cause for concern. Ultimately, this point can only be resolved through experiment, and so a synthesis of the cyclization precursor $\mathbf{7 5}$ was initiated.

Conversion of the commercially available benzoic acid derivative $\mathbf{7 0}$ into the tosylamide alkynylstannane 75 proceeded smoothly, Scheme 8 . The well-documented ${ }^{16}$ arene activating capabilities of oxazoline rings were exploited twice: first to activate the aryl core for nucleophilic aromatic substitution with Grignard reagent $\mathbf{7 2}$, and second to promote lateral metalation at the ortho aryl position as a means to facilitate attachment of the ethylamide side chain. Routine functional group manipulations completed the cyclization substrate synthesis. 


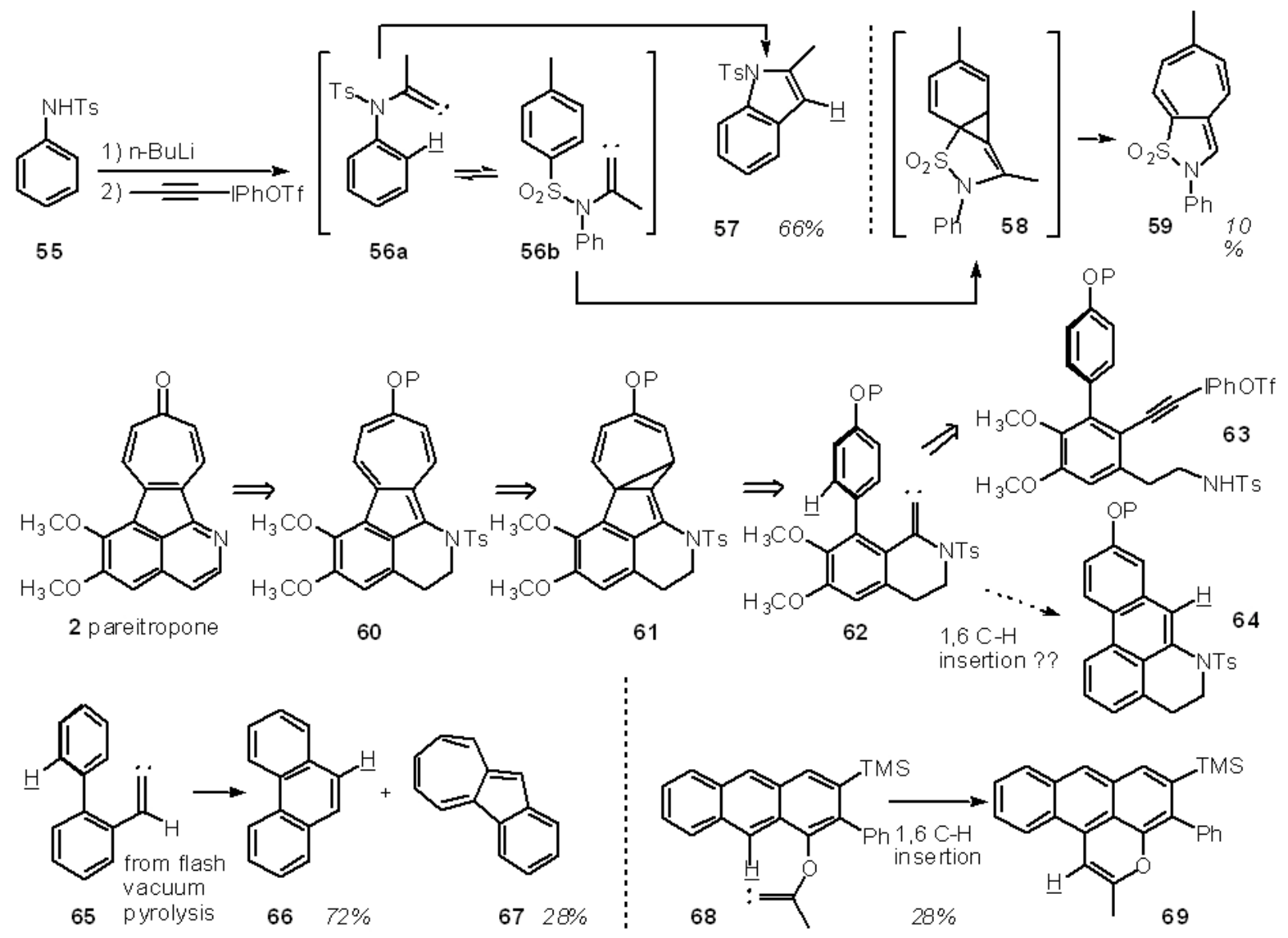

\section{Scheme 7}
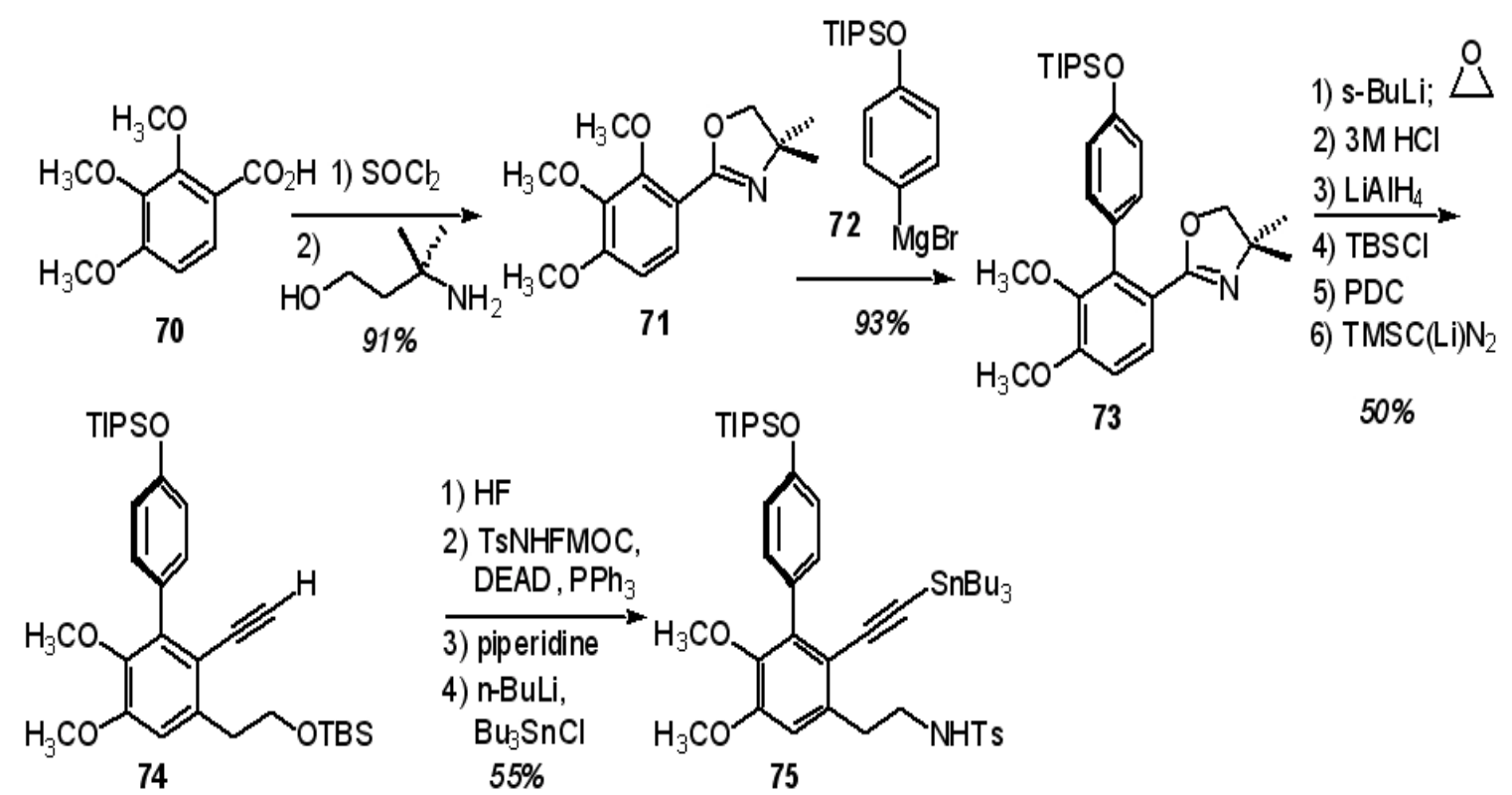

Scheme 8 
Transformation of alkynylstannane $\mathbf{7 5}$ into the unisolated iodonium salt $\mathbf{7 6}$, followed by treatment of this crude salt with strong base, initiated the key sequence of the synthesis route, Scheme 9. A single product (TLC) was rapidly formed. Spectroscopic characterization of this lone isolable product secured the structural assignment shown as 77. No products resembling phenanthrene 64 were isolated, or even detected, in the ${ }^{1} \mathrm{H}$ NMR spectrum of the crude reaction mixture. The selection for arene addition over 1,6 C-H insertion appeared complete. A rationale for this preference might be gleaned from the calculated (model) carbene structure $\mathbf{7 9},{ }^{17}$ which illustrates that the carbenic carbon is only $2.67 \AA$ from the peri-positioned aryl ring, whereas it is a full 2/3 $\AA$ more distant from the indicated C-H. Thus, reaction selectivity might simply emerge from proximity. Fluoride induced desilylation/desulfination was expected to deliver a dihydropareitropone product 78. Unfortunately, numerous trials with a range of acidic, neutral, or basic fluoride sources led exclusively to decomposition, even at $-78{ }^{\circ} \mathrm{C}$. No characterizable products, even in minute quantities, could be detected. Eventually, recourse to a two-phase system, $\mathrm{KF}$ on $\mathrm{Al}_{2} \mathrm{O}_{3}{ }^{18}$ provided the first glimmer of hope. Compound destruction was not observed - rather, a single product that did not display any aliphatic protons in its ${ }^{1} \mathrm{H}$ NMR spectrum, as expected for 78, was isolated following chromatography. Comparison of this product's spectroscopic data with those of pareitropone itself indicated identity! Apparently, aerobic oxidation of any first-formed dihydropareitropone $\mathbf{7 8}$ was rapid. In a few subsequent trials, examination of the crude reaction product by ${ }^{1} \mathrm{H}$ NMR spectroscopy suggested the presence of variable amounts of the dihydro product 78. Exposure of these NMR solutions to air led to an ${ }^{1} \mathrm{H}$ NMR spectrum identical to that seen in the crude pareitropone-forming runs. Further exploration of this unusual desilylation reagent led to the following observations: 1) $\mathrm{Al}_{2} \mathrm{O}_{3}$ alone did not affect 77; 2) KF suspended in THF destroyed 77; 3) $\mathrm{Bu}_{4} \mathrm{NF}$ in THF at $-78{ }^{\circ} \mathrm{C}$ destroyed pareitropone. These facts are consistent with a scenario wherein fluoride rapidly generates $\mathbf{7 8}$ (and then 2), but one (or both) of these species is susceptible to degradation by excess fluoride. $\mathrm{Al}_{2} \mathrm{O}_{3}$ may simply sequester $\mathbf{7 8}$ (and/or 2) by absorption, hence removing it from further exposure to fluoride. 


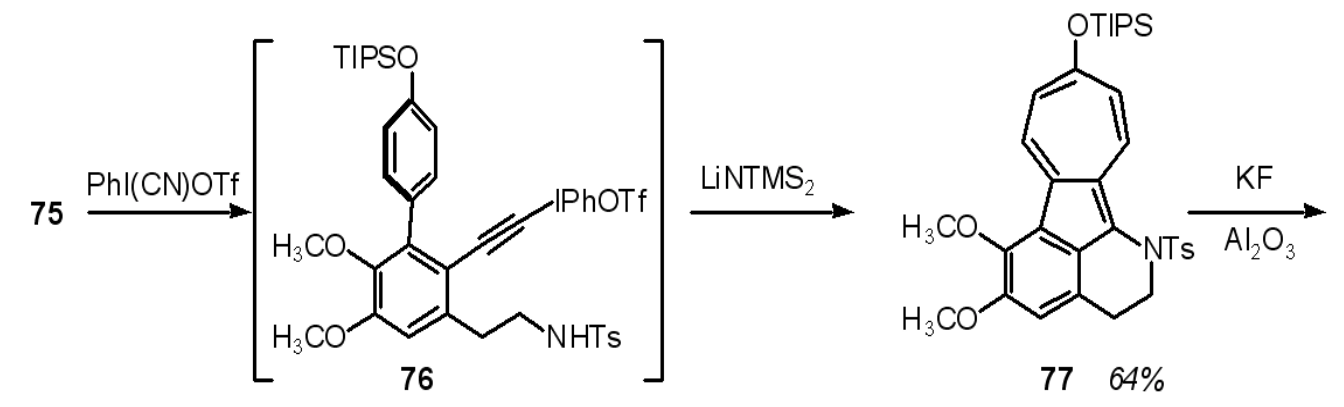

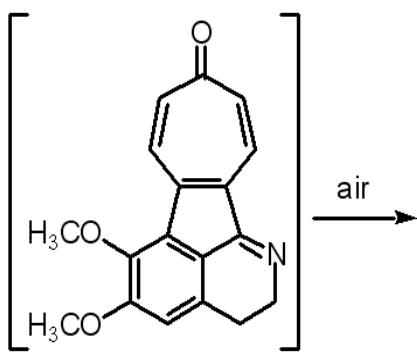

78

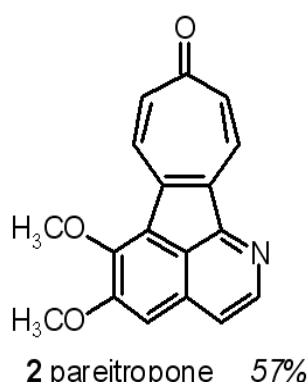

2 pareitropone $57 \%$

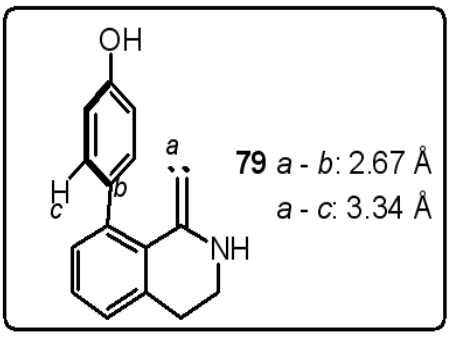

\section{Scheme 9}

In summary, the successful completion of the total syntheses of the alkaloids agelastatin A and pareitropone through alkynyliodonium salt chemistry emphasizes the value of these twocarbon conjunctive reagents in complex molecule synthesis. The ability to generate and selectively manipulate these iodonium salt intermediates in functionally rich environments remains a virtue of this chemistry. Their role in converting relatively simple substrates to much more structurally intricate products recommends them for strategy-level consideration in synthesis design.

\section{Acknowledgments}

Financial support from the National Institutes of Health under the auspices of GM 37681 is gratefully acknowledged. The unflagging efforts of Timothy Cutarelli, Dr. Romina Di Florio, Dr. Paul Hawkins, Dr. Pamela Mingo, Joe Saunders and Dr. Michelle Laci Wrobleski are acknowledged as well.

\section{References}

1. (a) Feldman, K. S.; Saunders, J. C.; Wrobleski, M. L. J. Org. Chem. 2002, 67, 7096. (b) Feldman, K. S.; Cutarelli, T. D.; Di Florio, R. J. Org. Chem. 2002, 67, 8528. 
2. (a) Stang, P. J.; Zhdankin, V. V. Tetrahedron 1998, 54, 10927. (b) Varvoglis, A. Tetrahedron 1997, 53, 1179.

3. (a) Varvoglis, A., Hypervalent Iodine in Organic Synthesis; Academic Press: London, 1997.

(b) Varvoglis, A. The Organic Chemistry of Polycoordinated Iodine; VCH Publishers: New York, 1992.

4. Varvoglis, A.; Spyroudis, S. Synlett 1998, 221.

5. Stang, P. J.; Zhdankin, V. V. J. Am. Chem. Soc. 1991, 113, 4571.

6. Ochiai, M.; Kunishima, M.; Nagao, Y.; Fuji, K.; Shiro, M.; Fujita, E. J. Am. Chem. Soc. 1986, 108, 8281.

7. Kirmse, W. Angew. Chem., Int. Ed. 1997, 36, 1164, and references cited therein.

8. (a) Stang, P. J. Chem. Rev. 1978, 78, 383, and references cited therein. (b) Ervin, K. M.; Ho, J.; Lineberger, W. C. J. Chem. Phys. 1989, 91, 5974. (c) Bodor, N.; Dewar, M. J. S.; Wasson, J. S. J. Am. Chem. Soc. 1972, 94, 9095. (d) Gallo, M. M.; Hamilton, J. P.; Schaeffer, H. F. III J. Am. Chem. Soc. 1990, 112, 8714. (e) Gilbert, J. C.; Giamalva, D. H. J. Org. Chem. 1992, 57, 4185. (f) Hayes, R. L.; Fattal, E.; Govind, N.; Carter, E. A. J. Am. Chem. Soc. 2001, 123, 641. (g) Reed, S. C.; Capitosti, G. J.; Zhu, Z.; Modarelli, D. A. J. Org. Chem. 2001, 66, 287. (h) Maercker, A. Angew. Chem., Int. Ed. 1993, 32, 1023.

9. Feldman, K. S.; Mingo, P. A.; Hawkins, P. C. D. Heterocycles 1999, 51, 1283.

10. Fujisawa, T.; Nagai, M.; Koike, Y.; Shimizu, M. J. Org. Chem. 1994, 59, 5865.

11. Ochiai, M.; Kunishima, M.; Tani, S.; Nagao, Y. J. Am. Chem. Soc. 1991, 113, 3135.

12. Brimble, M. A.; Brimble, M. T.; Hodges, R.; Lane, G. A. Aust. J. Chem. 1988, 41, 1583.

13. Feldman, K. S.; Bruendl, M. M.; Schildknegt, K.; Bohnstedt, A. C. J. Org. Chem. 1996, 61, 5440.

14. Brown, R. F. C.; Eastwood, F. W.; Harrington, K. J.; McMullen, G. L. Aust. J. Chem. 1974, 27, 2393.

15. Feldman, K. S.; Perkins, A. L. Tetrahedron Lett. 2001, 42, 6031.

16. (a) Meyers, A. I.; Gant, T. G. Tetrahedron 1994, 50, 2297. (b) Reuman, M.; Meyers, A. I. Tetrahedron 1985, 41, 837.

17. The model exocyclic alkylidenecarbene 79 was examined in Spartan 5.0 using a density functional calculation with the $\mathrm{DN}^{*}$ basis set and a perturbative Becke-Perdew approach.

18. (a) Blass, B. E.; Harris, C. L.; Portlock, D. E. Tetrahedron Lett. 2001, 42, 1611. (b) Blass, B. E. Tetrahedron 2002, 58, 9301. 